\title{
Microwave Photonic Hilbert Transformer Based on a Single Passband Microwave Photonic Filter for Simultaneous Channel Selection and Signal Processing
}

\author{
Jiejun Zhang, Student Member, IEEE, Weilin Liu, Student Member, IEEE, Fanqi Kong, Student Member, IEEE, \\ and Jianping Yao, Fellow, IEEE, Fellow, OSA
}

\begin{abstract}
A microwave photonic Hilbert transformer (MPHT) with a single passband and wide frequency tunable range based on a single passband microwave photonic filter (MPF) is proposed and experimentally demonstrated. The MPF is implemented using a broadband light source and a Mach-Zehnder interferometer (MZI) incorporating a phase modulator in one arm and a phaseshifted chirped fiber Bragg grating in the other arm. The optical signal at the output of the MZI is sent to a dispersion compensating fiber, and then, detected at a photodetector. The overall operation corresponds to a single passband MPF with a $\pi$ phase-shift at the center of the passband, which has the frequency response corresponding to a microwave Hilbert transformer. The central frequency of the MPHT can be continuously tuned by tuning the length difference between the two arms of the MZI using a tunable delay line. A theoretical analysis is performed, which is verified by simulations and an experiment. In the experiment, an MPHT with a bandwidth of $1.70 \mathrm{GHz}$ and a continuously tunable frequency from 5 to $15 \mathrm{GHz}$ is demonstrated. The proposed MPHT can find applications in multi-channel microwave communications systems, where simultaneous channel selection and Hilbert transformation are needed.
\end{abstract}

Index Terms-Hilbert transformer, microwave photonic filter (MPF), microwave signal processing, single passband filter.

\section{INTRODUCTION}

A microwave Hilbert transformer, also known as a quadrature filter, is among the very important signal processing blocks for microwave signal processing. It is widely employed for applications such as microwave communications, radars, and modern instrumentation [1]-[3]. Hilbert transformers can be implemented electronically. For example, an electronic discretetime Hilbert transformer was proposed in [4], which was used to achieve single-sideband modulation. By using an integrated microwave delay-line filter, a Hilbert transformer operating at $10 \mathrm{GHz}$ was demonstrated [5]. Electronic Hilbert transformers

Manuscript received February 20, 2014; revised June 10, 2014 and June 20 2014; accepted June 23, 2014. Date of publication June 25, 2014; date of current version August 11,2014. This work was supported by the Natural Sciences and Engineering Research Council of Canada. The work of J. Zhang was supported by a scholarship from the China Scholarship Council.

The authors are with the Microwave Photonics Research Laboratory, School of Electrical Engineering and Computer Science, University of Ottawa, Ottawa, ON K1N 6N5, Canada (e-mail: jpyao@eecs.uottawa.ca).

Color versions of one or more of the figures in this paper are available online at http://ieeexplore.ieee.org.

Digital Object Identifier 10.1109/JLT.2014.2333513 were also demonstrated for edge and corner detection of digital images [6], [7]. Hilbert transformers can also be implemented optically. In general, there are two types of photonic Hilbert transformers. The first type are used for the processing of optical signals, and simply called all-optical Hilbert transformers. The second type are used to process microwave signals, and are usually called microwave photonic Hilbert transformers (MPHT). An all-optical Hilbert transformer for processing optical signals can be implemented using an optical notch filter such as a fiber Bragg grating (FBG) [8]-[10], an optical interferometer [11], or an integrated optical ring resonator [12]. In an all-optical Hilbert transformer, the wavelength of the optical carrier is placed exactly at the notch of the filter, and the Hilbert transformation of the optical signal is achieved thanks to a $\pi$ phase shift at the notch, to introduce $\pi / 2$ to the negative spectral components and $-\pi / 2$ to the positive spectral components. Usually, an alloptical Hilbert transformer can have a bandwidth as large as several tens or hundreds of gigahertz or even terahertz [13]. However, an all-optical Hilbert transformer cannot be directly used for microwave signal processing, since the $\pi$ phase shift is introduced to the optical spectrum rather than the microwave spectrum, although the microwave signal to be processed is modulated on an optical carrier.

To realize an MPHT that can process microwave signals in the optical domain, the Hilbert transformer must be designed to have a microwave frequency response that corresponds to a microwave Hilbert transformer [14]-[17]. In [14], a four-tap delay line microwave photonic filter (MPF) was demonstrated for the implementation of an MPHT. Its application for instantaneous frequency measurement was presented in [15]. The system in [14] and [15] is relatively complicated and costly due to the use of two MZMs and a wideband microwave divider. A simplified MPHT was recently demonstrated using a single polarization modulator (PolM) [16] and a delay-line structure with nonuniformly spaced taps [17]. The major limitation of the MPHT in [14]-[17] is that multiple wavelengths corresponding to the multiple taps are required. To implement such an MPHT, the wavelength spacing should be tunable, making the system very complicated and costly. In addition, the spectral response of such an MPHT is periodic. For many applications, such as multi-channel microwave communications, an MPHT with a single passband is needed. 
Recently, a single passband MPF based on a broadband optical source and a Mach-Zehnder interferometer (MZI) was proposed [18]. The central frequency of the passband can be tuned by changing the length difference between the two arms in the MZI and the shape of the passband can be controlled by the tailoring the spectrum shape of the optical source. The concept has been improved to avoid dispersion-induced power penalty by placing the modulator in one arm of the MZI [19]. In addition, the frequency response of the filter can be controlled to have a quadratic phase response or a narrow passband by adding an optical phase filter, such as a chirped fiber Bragg grating (CFBG) [20] or a programmable optical filter [21] in one arm of the MZI. Therefore, by using a proper optical phase filter in the MZI, we are able to achieve an MPF with a spectral response corresponding to a microwave Hilbert transformer.

In this paper, we propose and demonstrate an MPHT with a single passband and a wideband frequency tunable range based on a single passband MPF. The MPF consists of a broadband optical source, an MZI incorporating a phase modulator (PM) in one arm and a phase-shifted chirped fiber Bragg grating (PSCFBG) in the other arm, a dispersive fiber, and a photodetector (PD). The key to implement an MPHT is to introduce a $\pi$ phase shift to the center of the passband, which is realized using the PS-CFBG in the MZI, to translate the phase jump at the notch of the PS-CFBG to the passband of the MPF. The bandwidth of the MPHT is determined by the bandwidth of the broadband source and the dispersion of the PS-CFBG. The central frequency of the MPHT can be tunable by changing the length difference between the two arms of the MZI. A theoretical study is performed, which is verified by simulations and an experiment. In the experiment, an MPHT with a bandwidth of $1.70 \mathrm{GHz}$ and a continuously frequency tunable range from 5 to $15 \mathrm{GHz}$ is demonstrated.

\section{PRINCIPLE}

The frequency response of an MPHT can be expressed as

$$
H_{\mathrm{HT}}(\Omega)= \begin{cases}e^{-j \pi / 2}, & \Omega<\Omega_{0} \\ e^{j \pi / 2}, & \Omega>\Omega_{0}\end{cases}
$$

where $\Omega$ is the microwave angular frequency and $\Omega_{0}$ is the frequency of the microwave carrier. As can be seen from (1), the MPHT has a unity magnitude response over an infinite bandwidth and a $\pi$ phase jump at $\Omega=\Omega_{0}$. For a practical MPHT, the bandwidth should be finite. Thus, there is a bandwidth in which (1) is satisfied, and the bandwidth is defined as the bandwidth of the microwave Hilbert transformation. For many applications, this band-limited feature is important since the MPHT can not only perform Hilbert transformation, but also bandpass filtering.

The schematic for the proposed MPHT is shown in Fig. 1. A broadband lightwave from an amplified spontaneous emission (ASE) source is coupled into an MZI through an optical coupler (OC1). In the upper arm of the MZI, a tunable optical delay line (TDL) is incorporated to change the length difference between the two arms of the MZI, and a PS-CFBG operating in the reflection mode through an optical circulator is connected in the arm. The PS-CFBG will provide a $\pi$ phase-shift at its central wavelength of the notch. In the lower arm of the MZI, a

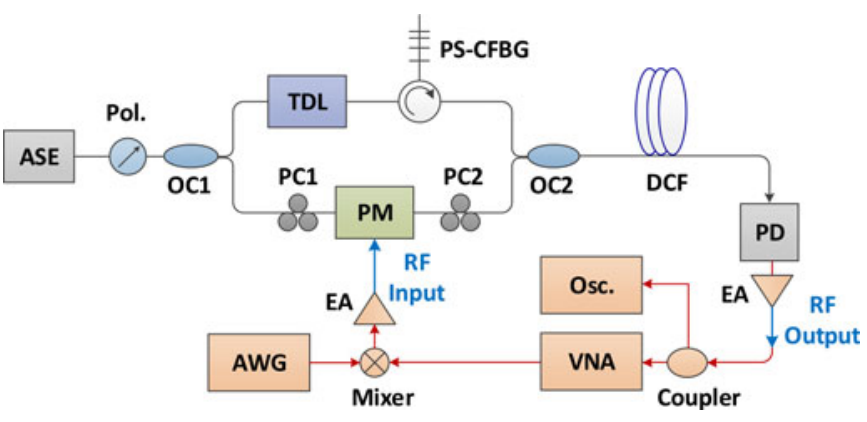

Fig. 1. Schematic of the proposed MPHT with a single passband. ASE: amplified spontaneous emission; Pol.: polarizer; OC: optical coupler; TDL: tunable delay line; PS-CFBG: phase-shifted chirped fiber Bragg grating; PC: polarization controller; PM: phase modulator; DCF: dispersion compensating fiber; PD: photodetector; EA: electrical amplifier; Osc.: oscilloscope; VNA: vector network analyzer; AWG: arbitrary waveform generator. The black lines and red lines represent optical and electrical link respectively. The blue arrows show the input and output ports of the MPHT.

PM is incorporated to which a microwave signal to be Hilbert transformed is applied. After being combined at the OC2, the lightwave from the MZI travels through a length of dispersion compensating fiber (DCF), and then, detected at the PD. The input and output ports of the MPHT are shown in the figure. A vector network analyzer (VNA) is used to measure the frequency response of the MPHT. An arbitrary waveform generator (AWG) and a real-time oscilloscope are used to generate the signal to be processed and sample the output signal, respectively.

Since the PS-CFBG has a quadratic phase response within its reflection band and a $\pi$ phase-shift at its central wavelength of the notch [22], the optical spectrum after travelling through the upper arm of the MZI can be written as

$$
E_{1}(\omega)=\frac{\sqrt{2}}{2} E_{0}(\omega) e^{j\left[\omega \Delta t+\frac{\beta_{F}\left(\omega-\omega_{0}\right)^{2}}{2}+\varphi(\omega)\right]}
$$

where $E_{0}(\omega)$ is the spectrum of the ASE source, $\omega$ is the angular frequency of the optical wave, $\Delta t$ is the time delay difference between the two arms of the MZI, which can be controlled by tuning the TDL, $\beta_{F}$ is the dispersion coefficient of the PSCFBG, $\omega_{0}$ is the central angular frequency of the PS-CBFG, and $\varphi(\omega)$ is the phase change introduced by the PS-CFBG. It is known that for a $\pi$-phase-shifted CFBG, we have $\varphi(\omega)=0$ for $\omega<\omega_{0}$, and $\varphi(\omega)=\pi$ for $\omega>\omega_{0}$ [22]. When no microwave signal is applied to the PM, the optical spectrum after travelling through the lower arm of the MZI can be simply written as $E_{2}(\omega)=\sqrt{2} / 2 \cdot E_{0}(\omega)$. Then, $E_{1}(\omega)$ and $E_{2}(\omega)$ are combined at OC2, the total optical spectrum at the output of OC2 is given by

$$
E_{3}(\omega)=\frac{1}{2} E_{0}(\omega)\left\{1+e^{j\left[\omega \Delta t+\frac{\beta_{F}\left(\omega-\omega_{0}\right)^{2}}{2}+\varphi(\omega)\right]}\right\} .
$$

The transfer function of the MZI is then given by 


$$
\begin{aligned}
T(\omega) & =\left|\frac{E_{3}(\omega)}{E_{0}(\omega)}\right| \\
& =1+\cos \left[\omega \Delta t+\frac{\beta_{F}\left(\omega-\omega_{0}\right)^{2}}{2}+\varphi(\omega)\right] .
\end{aligned}
$$

Then, the lightwave is sent to the DCF with a dispersion coefficient of $\beta_{D}$ and detected at the PD. The system can be seen as an MPF with a frequency response given by [19], [20]

$$
\begin{aligned}
H(\Omega) & \propto \int_{\delta \omega} N(\omega) T(\omega) \times \exp \left[\frac{-j \Omega \beta_{D}\left(\omega-\omega_{0}\right)}{2}\right] d \omega \\
& =\int_{\delta \omega} N(\omega) \cos \left[\omega \Delta t+\frac{\beta_{F}\left(\omega-\omega_{0}\right)^{2}}{2}+\varphi(\omega)\right] \\
& \times \exp \left[-\frac{j \Omega \beta_{D}\left(\omega-\omega_{0}\right)}{2}\right] d \omega
\end{aligned}
$$

where $\Omega$ is again the angular frequency of the microwave signal applied to the PM; $N(\omega)$ and $\delta \omega$ are the optical power density and the bandwidth of the ASE source, respectively. The baseband response induced by the constant of 1 in (4) is ignored in (5). Assuming that the ASE source has a flat spectrum, it can be seen from (5) that the frequency response of the MPF should have a shape corresponding to the Fourier transformation of the MZI transfer function $T(\Omega)$ centered at

$$
\Omega_{C}=\Delta t / \beta_{D}
$$

which is proportional to the time delay difference between the two arms of the MZI. Here, $T(\omega)$ is a sinusoidal function with a linearly varying free-spectrum range (FSR) induced by the quadratic term of $\omega$ in (4), and a phase shift at its center represented by $\varphi(\omega)$. The single passband MPF proposed here can be seen as a finite impulse response (FIR) filter with a tap spacing approaching to zero thanks to the use of an ASE Source that has a continuous spectrum. Thus, the FSR of the filter is approaching to infinite, leading to a spectral response with a single passband. The $\pi$ phase shift at $\omega=\omega_{0}$ due to the PS-CFBG would introduce positive and negative taps to the short and long wavelength halves of the spectrum (separated at $\omega_{0}$ ), respectively, leading to a notch at the center of the magnitude response and a $\pi$ phase shift at the center of the phase response. This is a frequency response that corresponds to a single passband MPHT.

The bandwidth of the MPF can be calculated by [20]

$$
B=\left|\beta_{F} / \beta_{D}\right| B_{O}
$$

where $B_{O}$ is the bandwidth of the PS-CFBG. As can be seen, the bandwidth of the MPF is independent of the time delay difference between the two arms of the MZI.

Based on (5), the frequency response of the MPF is simulated. In the simulation, we choose the dispersion coefficient of the DCF $\beta_{D}=432 \mathrm{ps}^{2}$ and the bandwidth of the ASE source $\delta \omega=2 \pi \times 1 \mathrm{THz}$. First, the frequency response and the bandwidth of the MPF are studied. In this case, the length difference between the two arms of the MZI is set to be $8.4 \mathrm{~mm}$, which corresponds to a passband centered at $15 \mathrm{GHz}$. Two different dispersion coefficients of the PS-CFBG at two values of 0 and
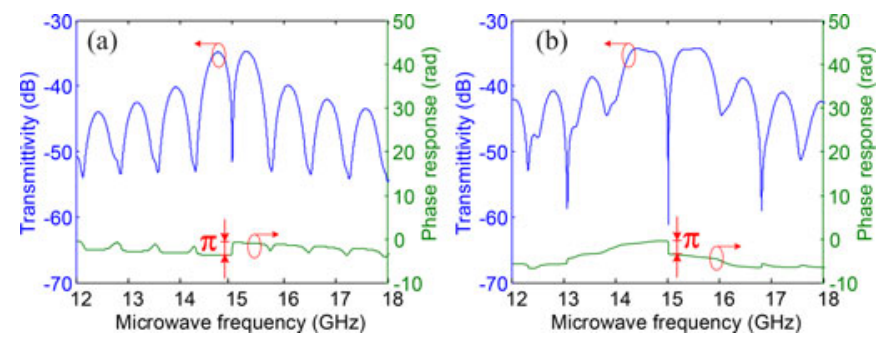

Fig. 2. Simulated magnitude (blue) and phase (green) response for the proposed MPHT with the PS-CFBG having a dispersion coefficient at two values of (a) $0 \mathrm{ps}^{2}$ and (b) $0.6 \mathrm{ps}^{2}$.

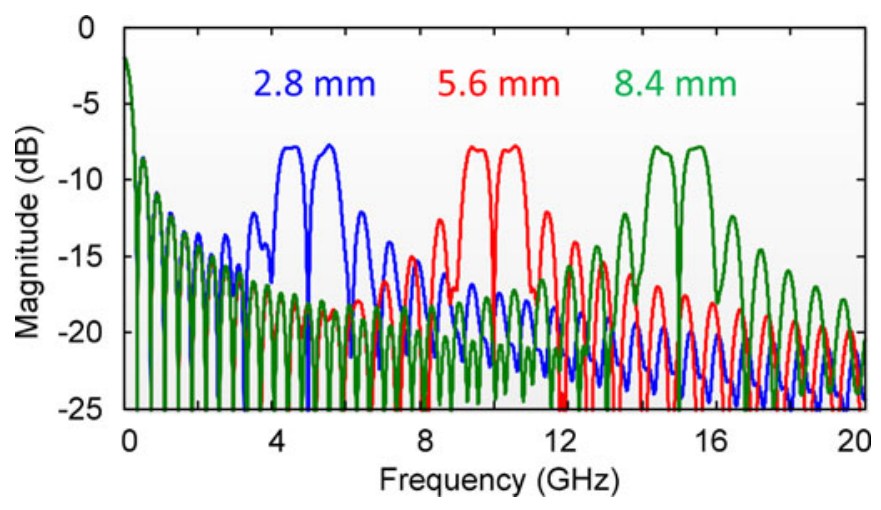

Fig. 3. Simulation for frequency tunability. The frequency responses of the proposed MPHT when the length difference between the two arms of the MZI is tuned at three values of $2.8,5.6$ and $8.4 \mathrm{~mm}$.

$0.6 \mathrm{ps}^{2}$ are considered. The simulation results are shown in Fig. 2(a) and (b), corresponding to the two different dispersion coefficients. As can be seen a passband with a notch at the center is achieved. A $\pi$ phase-shift at the notch frequency can also be seen. The simulation results confirm that the MPF has a frequency response that is required to achieve a singlepassband MPHT. In addition, by comparing Fig. 2(a) and (b), we can see that by changing the value of $\beta_{F}$, the bandwidth of the passband (i.e., the bandwidth of the MPHT) can be changed. This feature is important since an MPHT with different bandwidths may be needed for different applications. For example, in a multi-channel microwave communication system, an MPHT with bandwidth close to the channel spacing should be used. In the simulation, when $\beta_{F}=0.6 \mathrm{ps}^{2}$, the MPHT has an operational bandwidth of around $1.70 \mathrm{GHz}$, which is suitable for applications in a multi-channel microwave communication system, where the channel spacing is about $1.70 \mathrm{GHz}$.

Then, the frequency tunability of the MPHT is investigated. To do so, we change the length difference between the two arms of the MZI by tuning the TDL. The simulated magnitude response is shown in Fig. 3. In the simulation, the dispersion coefficient for the DCF is set at $\beta_{F}=0.6 \mathrm{ps}^{2}$ and the MZI length difference is tuned at 2.8, 5.6, and $8.4 \mathrm{~mm}$. A single passband MPF with a notch at the center is obtained. The central frequency of the filter varies linearly as a function of the length difference, as predicted by (6). For the three length difference values of 2.8, 5.6, and $8.4 \mathrm{~mm}$, the center frequencies of the MPF 
are 5, 10, and $15 \mathrm{GHz}$, respectively. As the bandwidth of the MPF is only decided by the optical spectral bandwidth and dispersion coefficients of the PS-CFBG and DCF according to (7), the shape of the frequency response is maintained unchanged when the central frequency of the MPF is tuned. This is different from an MPHT based on a delay-line structure, where the frequency tuning would lead to the change of the shape of the frequency response [16], [17]. This is another important feature that is highly required for many applications.

\section{EXPERIMENT}

An experiment based on the setup shown in Fig. 1 is performed to evaluate the operation of the proposed singlepassband MPHT. The system consists of an ASE source, an MZI incorporating a PM in one arm and a TDL and a programmable optical filter (POF) in the other arm, a DCF, and a PD. A broadband lightwave from the ASE source is sent to the MZI via a $1 \times 2$ optical coupler $(\mathrm{OC} 1)$. Since the PM in the lower arm only supports linear polarization along one direction, to minimize the polarization-dependent loss, the lightwave from the ASE source is linearly polarized by a polarizer before it is launched into the MZI, and sent to the PM via a polarization controller (PC1). A POF (Finisar WaveShaper 4000S) in the upper arm instead of the PS-CFBG is used. It is configured to function as the PS-CFBG, with a passband of $1 \mathrm{THz}$ wide and a quadratic phase response in the passband. In addition, a $\pi$ phase-shift at the central wavelength of $1550 \mathrm{~nm}$ is also incorporated. A VNA (Agilent E8364A) is connected to the PD via an electrical amplifier (EA1) to measure the frequency response of the MPHT. A microwave signal generated by mixing a waveform from an AWG (Tektronix AWG7102) with a microwave carrier from the VNA at a mixer is then applied to the PM after amplification by an electrical amplifier (EA2). A real-time oscilloscope (Agilent 93204A) is also connected at the output of the PD to record the temporal waveform from the MPHT.

We first measure the frequency response of the MPHT. To do so, the AWG is set to generate a sequence of "1" bit (high voltage level). The TDL is tuned such that the length difference between the two arms of the MZI corresponds to a central frequency of $15 \mathrm{GHz}$. The quadratic phase of the POF is set to be $\beta_{F}=0.6 \mathrm{ps}^{2}$, a value identical to that used in the simulation. The measured frequency response of the MPHT is shown in Fig. 4. A flat-top microwave filter at $15 \mathrm{GHz}$ with a bandwidth of $1.70 \mathrm{GHz}$ and a notch of $300 \mathrm{MHz}$ is achieved. In the phase response, a $\pi$ phase-shift is observed at the center of the notch. The linear phase response of the link is calibrated in the measurement. It can be seen that the measured result is in good agreement with the simulation results.

Then, the frequency tunability is studied. The central frequency of the MPHT is tuned by tuning the TDL. As shown in Fig. 5, when the length difference is tuned from 2.8 to $8.4 \mathrm{~mm}$, the central frequency of the MPHT is changed from 5 to $15 \mathrm{GHz}$, while the shape of the filter response is maintained unchanged. Again, the experimental results agree well with the simulation results shown in Fig. 3. Since the MPHT has large frequency tunability, it can be used to not only perform microwave Hilbert

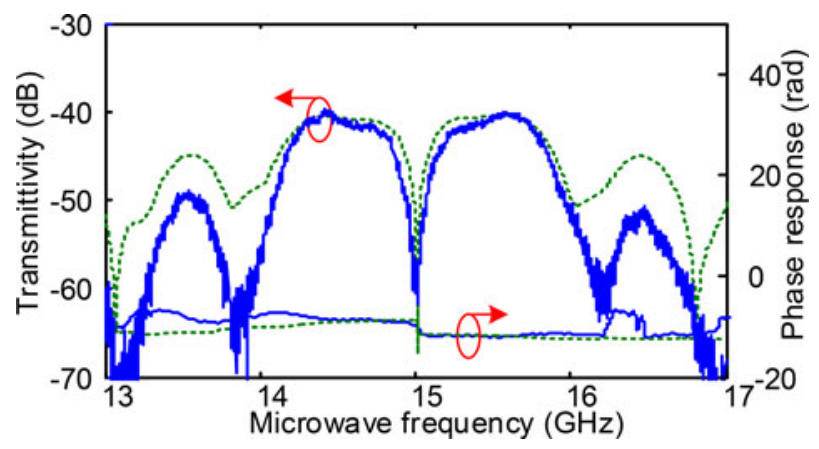

Fig. 4. Measured magnitude and phase responses of the MPHT (blue-solid). For Comparison, the simulated results (green-dotted) in Fig. 2(b) are also included.

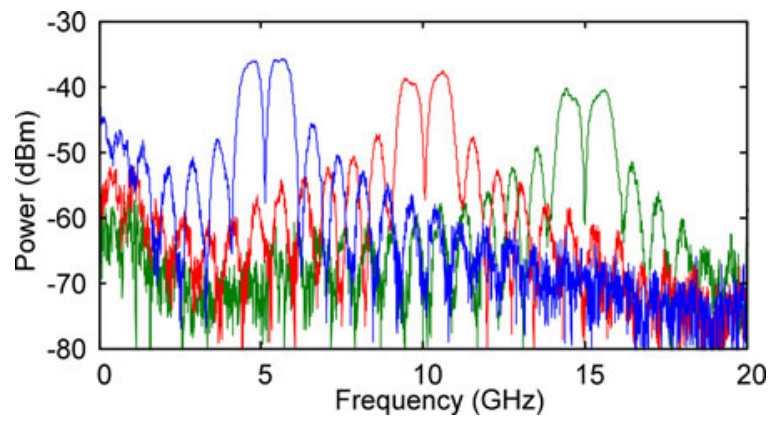

Fig. 5. Frequency tuning of the MPHT.

transformation but also do microwave filtering, to select the channel of interest in a multi-channel microwave communications system.

The MPHT is then used to realize real-time Hilbert transformation of a microwave signal. An electrical Gaussian pulse train with a repetition rate of $20 \mathrm{MHz}$ is generated by the AWG. The full-width at half-maximum of an individual pulse in the pulse train is $3.2 \mathrm{~ns}$. A microwave carrier generated by the VNA is mixed with the Gaussian pulse train and sent to the PM. The central frequency of the MPHT is tuned by tuning the TDL to be identical to the frequency of the microwave carrier. The signal at the output of the MPHT is sampled by the OSC. In the experiment, the microwave carrier frequency of the microwave carrier is tuned at 7.3 and $8.0 \mathrm{GHz}$. Due to the limited bandwidths of the EAs (EA1 and EA2) and the mixer, no other frequencies are tested.

First, the MPHT is configured to operate at $7.3 \mathrm{GHz}$. The Gaussian pulse train modulated on the $7.3 \mathrm{GHz}$ microwave carrier is applied to the PM. Fig. 6(a) shows the waveform at the output of the PD. As can be seen the Gaussian pulse is Hilbert transformed. The signal-to-noise ratio (SNR) of the signal is relatively poor because of the high noise from the ASE source, and the noises from the PM and the PD.

Since the Gaussian waveform is modulated on a microwave carrier, we may get the Hilbert transformed Gaussian waveform by down-converting the signal at the output of the PD by mixing the signal with the same microwave carrier and then using a low-pass filter. Fig. 6(b) shows the down-converted waveform 

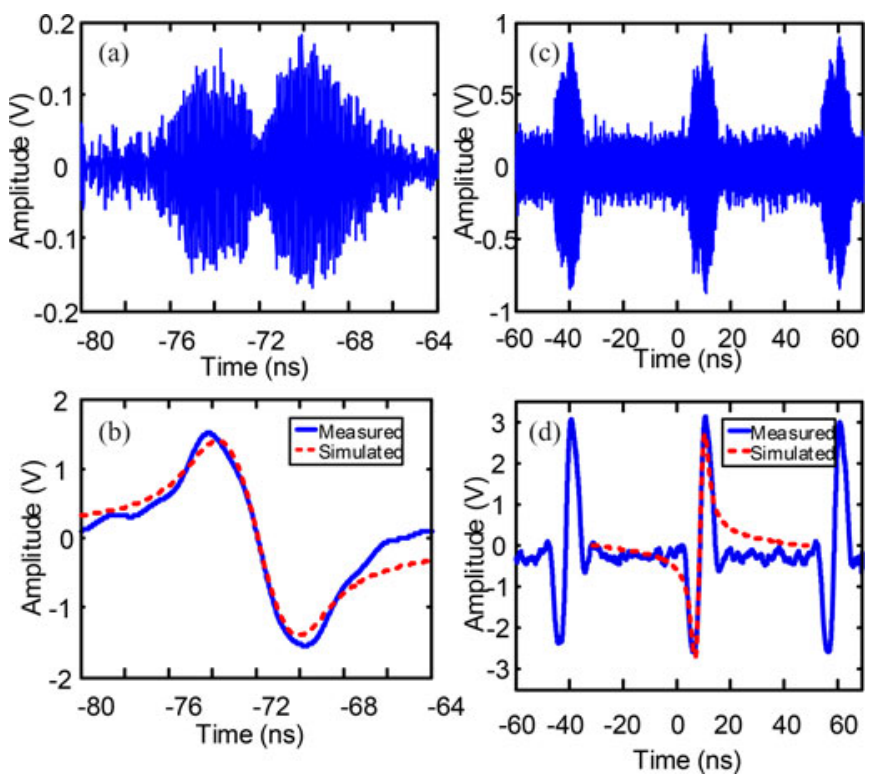

Fig. 6. Experimental results: (a) the Hilbert transformed waveform at the output of the PD when the carrier frequency is tuned at $7.3 \mathrm{GHz}$, (b) the measured Hilbert transformed Gaussian pulse (blue-solid) and the ideally Hilberttransformed Gaussian pulse (red-dotted). (c) The Hilbert transformed waveform at the output of the PD when the carrier frequency is tuned at $8.0 \mathrm{GHz}$, (d) the measured Hilbert transformed Gaussian pulse (blue-solid) and the ideally Hilbert-transformed Gaussian pulse (red-dotted).

(solid line). A theoretically calculated waveform corresponding to an ideal Hilbert-transformed Gaussian pulse is also shown for comparison (dashed line). As can be seen, a good agreement is reached.

Then, the microwave carrier frequency is tuned to $8.0 \mathrm{GHz}$. To process the microwave signal at $8 \mathrm{GHz}$, the center frequency of the MPHT is also tuned at $8 \mathrm{GHz}$, which is done by tuning the length difference at $4.48 \mathrm{~mm}$ by tuning the TDL. The waveform at the output the MPHT is again captured by the OSC, which is shown in Fig. 6(c). Again, to show the Hilbert-transformed Gaussian pulse, the signal at the output of the PD is mixed with the same microwave carrier at $8 \mathrm{GHz}$ and filtered by a low-pass filter, with the result shown in Fig. 6(d) (solid line). An ideal Hilbert transformed Gaussian pulse is also shown (dashed line). Again, a good agreement is reached.

\section{CONCLUSION}

We have proposed and demonstrated a microwave Hilbert transformer with a single passband and a wideband frequency tunable range based on an MPF. The MPF was implemented using a broadband light source, an MZI incorporating a PM in one arm and $\mathrm{PS}-\mathrm{CFBG}$ in the other arm, a DCF, and a PD, which had a frequency response corresponding to a single-passband MPHT. The single passband was realized due to the use of a broadband light source. The frequency tunability was realized by tuning the time delay difference between the two arms of the MZI. A theoretical analysis was performed, which was verified by simulations and an experiment. In the experiment, an MPHT with a bandwidth of $1.70 \mathrm{GHz}$ and a continuously tunable frequency from 5 to $15 \mathrm{GHz}$ was demonstrated. The use of the MPHT to perform Hilbert transformation of a microwave signal with a carrier frequency at 7.3 and $8.0 \mathrm{GHz}$ was demonstrated. The proposed MPHT can find applications in multi-channel microwave communication systems where the signal processors are expect to perform signal processing as well as channel selection.

\section{REFERENCES}

[1] A. D. Poularikas, Transforms and Applications Handbook. Boca Raton, FL, USA: CRC Press, 2010.

[2] A. I. Zayed and A. G. Garcia, "New sampling formulae for the fractional Fourier transform," Signal Process., vol. 77, no. 1, pp. 111-114, Aug. 1999.

[3] V. Cizek, "Discrete Hilbert transform," IEEE Trans. Audio Electroacoust., vol. AE-18, no. 4, pp. 340-343, Dec. 1970.

[4] C. C. Tseng and S. C. Pei, "Design and application of discrete-time fractional Hilbert transformer," IEEE Trans. Circuits Syst. II, Analog Digit. Signal Process., vol. 47, no. 12, pp. 1529-1533, Dec. 2000.

[5] C. D. Holdenried, J. W. Haslett, and B. Davies, "A fully integrated 10$\mathrm{Gb} / \mathrm{s}$ tapped delay Hilbert transformer for optical single sideband," IEEE Microw. Wireless Compon. Lett., vol. 15, no. 5, pp. 303-305, May 2005.

[6] S. C. Pei and M. H. Yeh, "Discrete fractional Hilbert transform," IEEE Trans. Circuits Syst. II, Analog Digit. Signal Process., vol. 47, no. 11, pp. 1307-1311, Nov. 2000.

[7] K. Kohlmann, "Corner detection in natural images based on the 2-D Hilbert transform," Signal Process., vol. 48, no. 3, pp. 225-234, Feb. 1996.

[8] M. H. Asghari and J. Azaña, "All-optical Hilbert transformer based on a single phase-shifted fiber Bragg grating: Design and analysis," Opt. Lett., vol. 34, no. 3, pp. 334-336, Jan. 2009.

[9] M. Li and J. Yao, "Experimental demonstration of a wideband photonic temporal Hilbert transformer based on a single fiber Bragg grating," IEEE Photon. Technol. Lett., vol. 22, no. 21, pp. 1559-1561, Nov. 2010.

[10] H. Shahoei and J. P. Yao, "Continuously tunable fractional Hilbert transformer by using a single $\pi$-phase shifted FBG," IEEE Photon. Technol. Lett., vol. 25, no. 22, pp. 2225-2228, Nov. 2013.

[11] K. Takano, N. Hanzawa, S. Tanji, and K. Nakagawa, "Experimental demonstration of optically phase-shifted SSB modulation with fiber-based optical Hilbert transformers," presented at the Optical Fiber Communication Conf., Anaheim, CA, USA, 2007, Paper JThA48.

[12] L. Zhuang, M. R. Khan, W. Beeker, A. Leinse, R. Heideman, and C. Roeloffzen, "Novel microwave photonic fractional Hilbert transformer using a ring resonator-based optical all-pass filter," Opt. Exp., vol. 20, no. 24, pp. 26499-26510, Nov. 2012.

[13] R. Ashrafi and J. Azaña, "Terahertz bandwidth all-optical Hilbert transformers based on long-period gratings," Opt. Lett., vol. 37, no. 13, pp. 2604-2606, Jul. 2012.

[14] H. Emami, N. Sarkhosh, L. A. Bui, and A. Mitchell, "Wideband RF photonic in-phase and quadrature-phase generation," Opt. Lett., vol. 33, no. 2, pp. 98-100, Jan. 2008.

[15] H. Emami, N. Sarkhosh, L. Bui, and A. Mitchell, "Amplitude independent RF instantaneous frequency measurement system using photonic Hilbert transform," Opt. Exp., vol. 16, no. 18, pp. 13707-13710, Aug. 2008.

[16] Z. Li, W. Li, H. Chi, X. Zhang, and J. P. Yao, "A continuously tunable microwave fractional Hilbert transformer based on a photonic microwave delay-line filter using a polarization modulator," IEEE Photon. Technol. Lett., vol. 23, no. 22, pp. 1694-1696, Nov. 2011.

[17] Z. Li, Y. Han, H. Chi, X. Zhang, and J. P. Yao, "A continuously tunable microwave fractional Hilbert transformer based on a nonuniformly-spaced photonic microwave delay-line filter," J. Lightw. Technol., vol. 30, no. 12, pp. 1948-1953, Jun. 2012.

[18] J. Mora, B. Ortega, A. Díez, J. L. Cruz, M. V. Andrés, J. Capmany, and D. Pastor, "Photonic microwave tunable single-bandpass filter based on a Mach-Zehnder interferometer," J. Lightw. Technol., vol. 24, no. 7, pp. 2500-2509, Jul. 2006.

[19] X. Xue, X. Zheng, H. Zhang, and B. Zhou, "Widely tunable singlebandpass microwave photonic filter employing a non-sliced broadband optical source," Opt. Exp., vol. 19, no. 19, pp. 18423-18429, Sep. 2011.

[20] X. Xue, X. Zheng, H. Zhang, and B. Zhou, "Tunable chirped microwave photonic filter employing a dispersive Mach-Zehnder structure," Opt. Lett., vol. 36, no. 17, pp. 3518-3520, Sep. 2011. 
[21] X. Xue, X. Zheng, H. Zhang, and B. Zhou, "High-Q single-bandpass photonic RF filter overcoming optical third-order dispersion based on programmable spectrum shaping of a broadband optical source," presented at the Optical Fiber Communication Conf., Anaheim, CA, USA, 2013, Paper OTu2H.2.

[22] T. Erdogan, "Fiber grating spectra," J. Lightw. Technol., vol. 15, no. 8, pp. 1277-1294, Aug. 1997.

Jiejun Zhang (S'12) received the B.Eng. degree in electronic science and technology from the Harbin Institute of Technology, Harbin, China, in 2010, and the M.Sc. degree in optical engineering from the Huazhong University of Science and Technology, Wuhan, China. He is currently working toward the $\mathrm{Ph} . \mathrm{D}$. degree in electrical engineering and computer science with the Microwave Photonics Research Laboratory, School of Electrical Engineering and Computer Science, University of Ottawa, Ottawa, ON, Canada.

His research interests include photonic generation of microwave waveforms, photonic processing of microwave signals, and fiber optic sensors.

Weilin Liu (S'10) received the B.Eng. degree in electronic information engineering from the University of Science and Technology of China, Hefei, China, in 2009 and the M.A.Sc. degree in electrical and computer engineering from the School of Electrical Engineering and Computer Science, University of Ottawa, Ottawa, ON, Canada, in 2011.

$\mathrm{He}$ is currently working toward the Ph.D. degree and working in the Microwave Photonics Research Laboratory, School of Electrical Engineering and Computer Science, University of Ottawa. His research interests include microwave/terahertz generation, optical signal processing, fiber Bragg grating, and their applications in microwave photonic systems.
Fanqi Kong (S'13) received the B.Eng. degree in optoelectronic information engineering from Huazhong University of Science and Technology, Wuhan, China, in 2012. He is currently working toward the M.A.Sc. degree in the Microwave Photonics Research Laboratory, School of Electrical Engineering and Computer Science, University of Ottawa, Ottawa, ON, Canada.

His current research interests include photonic generation of microwave signals and applications in sensing systems.

Jianping Yao (M'99-SM'01-F'12) is a Professor and the University Research Chair in the School of Electrical Engineering and Computer Science, University of Ottawa, Ottawa, ON, Canada. He received the Ph.D. degree in electrical engineering from the Universite de Toulon, Toulon, France, in December 1997. He joined the School of Electrical and Electronic Engineering, Nanyang Technological University, Singapore, as an Assistant Professor in 1998. In December 2001, he joined the School of Electrical Engineering and Computer Science, University of Ottawa, as an Assistant Professor, where he became a tenured Associate Professor in 2003, and a Full Professor in 2006. He became the University Research Chair in Microwave Photonics in 2007. From July 2007 to June 2010, he was the Director of the Ottawa-Carleton Institute for Electrical and Computer Engineering. He was re-appointed as the Director of the OttawaCarleton Institute for Electrical and Computer Engineering in 2013.

He has published more than 450 papers, including more than 260 papers in peer-reviewed journals and 190 papers in conference proceedings. He was a Guest Editor for the Focus Issue on Microwave Photonics in Optics Express in 2013 and a Feature Issue on Microwave Photonics in Photonics Research in 2014. He is currently a Topical Editor for Optics Letters, and serves on the Editorial Board of the IEEE TRANSACTIONS ON MiCROWAVE THEORY AND TECHNIQUES. He is a Chair of numerous international conferences, symposia, and workshops, including the Vice-TPC Chair of the 2007 IEEE Microwave Photonics Conference, TPC Co-chair of the 2009 and 2010 Asia-Pacific Microwave Photonics Conferences, TPC Chair of the high-speed and broadband wireless technologies subcommittee of the 2009-2012 IEEE Radio Wireless Symposia, TPC Chair of the microwave photonics subcommittee of the 2009 IEEE Photonics Society Annual Meeting, TPC Chair of the 2010 IEEE Microwave Photonics Conference, and General Co-Chair of the 2011 IEEE Microwave Photonics Conference. He received the 2005 International Creative Research Award at the University of Ottawa and the 2007 George S. Glinski Award for Excellence in Research. He was selected to receive an inaugural OSA outstanding reviewer award in 2012. He is an IEEE MTT-S distinguished microwave lecturer for 2013-2015.

Dr. Yao is a registered Professional Engineer of Ontario. He is a Fellow of the Canadian Academy of Engineering. 